\title{
The Comparison of Two Kinds of Extraction Methods of Circular Manual Mark in Visual Measurement
}

\author{
LIU Ze-qing ${ }^{1}$, HE Shu-ming ${ }^{2}$, ZHANG Yu-rong ${ }^{1}$ \\ ${ }^{1}$ Department of Artillery Engineering, Shijiazhuang Mechanical Engineering College, Shijiazhuang \\ 050003, P. R. China; \\ ${ }^{2}$ The Second Student Brigade, Shijiazhuang Mechanical Engineering College, Shijiazhuang \\ 050003, P. R. China;
} Keywords: Circular Marks; Image Extraction; Image Positing; Region of Interest (ROI); Ellipse
Fitting

\begin{abstract}
To solve the problem of extracting and locating the manual circular marks from complex background in the image of range visual measurement, two kinds of extraction methods of circular manual mark were studied, one was the interactive extraction method based on region of interest and the other was the extraction method based on image gray scale and geometric morphology. In this paper, the two methods are introduced at first, and then the methods are adopted to extract the circular marks from the image of visual measurement. The edge extraction and positioning results are compared, and the advantages and disadvantages of the two methods are analyzed. The result shows that the first method can extract the edge of the circular mark smoothly, but this method a lot by the image of the circular marks, while, the second method is less affected by the image of the circular mark. But the edge extracted by the second method is serrated, and part of the circular mark pixels misidentified as background, which is not in conformity with the actual situation.
\end{abstract}

\section{Introduction}

Setting the artificial sign as the known point are commonly used in visual measurement to calibrate the inside and outside parameters of camera, firstly, the world coordinate of artificial sign can be gained by measuring in advance, and the position coordinate of artificial sign on the image should be determined by image extraction.For the problems of long measuring distance, complex background, numerous interference factors in shooting range visual measurement, selecting the circular mark as seen in Fig. 1 as the marked point, the circular mark which has rotation invariance will be imaged as ellipse when shooting in certain angle, it is more stable when compared with linear or spot marks.In the situation of determined camera calibration model, the calibration accuracy of inside and outside parameters of camera is not only related to the world coordinate accuracy of marked point, but also it is related to the extracting and positioning accuracy of the marked point on the image.

The circular mark will be presented as ellipse owing to the angle of shooting, the extracting of circular mark mostly relates to the testing issue of the ellipse. The Hough transform and its modified algorithm[2,3] is a common ellipse testing method, the Hough transform method need to map the image space to parameter space in order to do multidimensional statistic calculation when carrying out the ellipse testing, the computation is huge, and the computation efficiency is low. Meanwhile, when carrying out the on-site visual measurement, the image background is more complex, and it also will be affected by complex factors such as shock wave, dust raising when carrying out static explosive experiment etc., so the employment of Hough transform is relatively difficult. To solve these problems, we have studied the interactive extraction method based on the region of interest and the extraction method based on image gray scale and geometric morphology to extract and position the circular mark image accordingly. The computational complexity of these two methods are relatively low, it will be able to complete the positioning of circular mark quickly in conditions of known marked point number. 


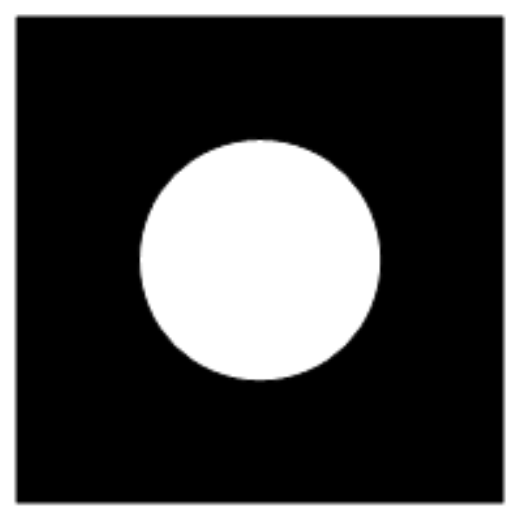

Fig.1 circular marked point

\section{Least squares edge fitting extracting method based on Region of Interest}

\subsection{The extracting of Region of Interest}

A Region of Interest

When extracting target in images which have complex background, the target extracting efficiency is relatively low, and the extracting effect is relatively bad. To reduce the searching range when extracting target and the effect of complex background factors, the extracting in region of interest where target is located may be done before target extracting. Region of interest(region of interesting, ROI) means the regions that can attract the attention of human optical system in the image, video, these regions often have more outstanding features like luminance, color, shape, texture etc. when compared with the surroundings. Region of interest includes the major information and key information of image, therefore, the treatment to region of interest may replace the treatment to the original image, this can reduce the computational complexity and the degree of complexity ${ }^{[5]}$ in image treating greatly.

The extracting methods of region of interest mostly comprise: human-machine interactive extracting method, method based on point of attention, method based on visual attention model, method based on specific object segmentation. Wherein, human-machine interactive method is easy to operate, and easy to realize, therefore using human-machine interactive method to extract the region of interest where calibrated circle being located.

1.1.2 The interactive extracting of Region of Interest

Herein, taking the region surrounded by the black edge outside the calibrated circle as the region of interest, the interactive extracting of the region of interest may be realized by artificially selecting the rectangle region where calibrated circle being located. Using the MATLAB2014b software as the image processing tool, and using waitforbuttonpress function to obtain the coordinate of position point while the mouse is clicking on the image to obtain the region being selected.

B The edge extracting of circular mark

Taking one circular mark among the others as a example to explain the extracting of circular marks, transforming the extracting of circular mark to the extracting of ellipse, the testing procedures of the ellipse are as follows:

(1) Inputting the region of interest I1 of the circular mark, recording the position coordinates of the top left corner of the region of interest;

(2) Filtering, median filtering the I1, and the influence to the edge of the ellipse by the noise may be reduced while the target information won't loss, and the connectivity between ellipse edge pixels may be enhanced;

(3) Binary processing, binary processing the image, set the background region pixel value as 0 , and set the circular mark region as 1 ;

(4) Region filling, region filling the interior of the enclosed region, and eliminating the hollow at the edge;

(5) Eliminating the jamming region, testing the connected region on the image, and accounting the pixel value of each connected region as the feature area, the largest area is the region that circular mark being located, eliminating the other regions whose areas are smaller; 
(6) Edge extracting, as the canny operator has the characteristic of high accuracy, high arithmetic speed, using canny operator to extract the edge of circular mark;

(7) According to the edge coordinates extracted in (6) and the position coordinates $\left(\mathrm{x}_{0}, \mathrm{y}_{0}\right)$ of the top left corner, the coordinates of the circular mark edge of the original image on the image can be obtained.

\section{Least squares edge fitting and the circular mark positing}

After edge extracting the circular mark, the position coordinates of all edge points can be obtained, using least squares edge fitting method to restore the shape of the circular mark. The general algebraic form of the ellipse may be represented as $a x^{2}+b x y+c y^{2}+d x+e y+f=0$, wherein $a, b \ldots f$ is the coefficients of the ellipse, the above formula may be rewrote to the matrix form, i.e.

$$
\left[\begin{array}{llllll}
x^{2} & x y & y^{2} & x & y & 1
\end{array}\right]\left[\begin{array}{c}
a \\
b \\
c \\
d \\
e \\
f+1
\end{array}\right]=1
$$

While the edge points $\left(x_{i}, y_{i}\right)$ of multiple ellipses are known, then,

$$
\begin{gathered}
{\left[\begin{array}{cccccc}
x_{1}^{2} & x_{1} y_{1} & y_{1}^{2} & x_{1} & y_{1} & 1 \\
x_{2}^{2} & x_{2} y_{2} & y_{2}^{2} & x_{2} & y_{2} & 1 \\
\vdots & \vdots & \vdots & \vdots & \vdots & \vdots \\
x_{i}^{2} & x_{i} y_{i} & y_{i}^{2} & x_{i} & y_{i} & 1
\end{array}\right]\left[\begin{array}{c}
a \\
b \\
c \\
d \\
e \\
f+1
\end{array}\right]=\left[\begin{array}{c}
1 \\
1 \\
\vdots \\
1
\end{array}\right]} \\
\operatorname{set} A=\left[\begin{array}{cccccc}
x_{1}^{2} & x_{1} y_{1} & y_{1}^{2} & x_{1} & y_{1} & 1 \\
x_{2}^{2} & x_{2} y_{2} & y_{2}^{2} & x_{2} & y_{2} & 1 \\
\vdots & \vdots & \vdots & \vdots & \vdots & \vdots \\
x_{i}^{2} & x_{i} y_{i} & y_{i}^{2} & x_{i} & y_{i} & 1
\end{array}\right],
\end{gathered}
$$

$X=\left[\begin{array}{llllll}a & b & c & d & e & f+1\end{array}\right]^{T}, B=\left[\begin{array}{llll}1 & 1 & \cdots & 1\end{array}\right]^{T}$

then formula(2) can be represented as

$$
A X=B
$$

according to least squares method,

$$
X=\left(\mathrm{A}^{\mathrm{T}} \mathrm{A}\right)^{-1} B
$$

According to $X, 6$ coefficients of the ellipse may be obtained, while the six feature coefficients of the ellipse are known, the outline of the ellipse may be fitted, and the center of the ellipse will be

$$
\left\{\begin{array}{l}
x_{c}=\frac{b e-2 c d}{4 a c-b^{2}} \\
y_{c}=\frac{b d-2 a e}{4 a c-b^{2}}
\end{array}\right.
$$

\section{The extracting method based on image gray scale and geometric morphology}

\subsection{The extracting of circular mark}

Preprocessing the image according to the gray scale feature of the image, then testing the edge, filling the enclosed outline, and the multiple regions including circular mark may be obtained, then eliminating the jamming region according to the area features of the circular mark. The specific processing procedures are as follows: 
(1) Median filtering, reducing the effect of the noise, and the edge may be more clear;

(2) Transforming the image gray scale, and the contrast of the bright and dark sections on the image may be increased;

(3) Edge testing the image, and multiple outlines including circular mark may be obtained;

(4) Hollow region filling, filling the interior of the region with enclosed outline, and the region with opened outline will stay the same;

(5) Eliminating the liner region on the image according to the circularity of the region, the circularity $C$ reflecting the extent of a ellipse being close to a circle, with its value near 1 , the shape of the region nears circle, the circularity is calculated according to $C=4 \pi S / L^{2}$, wherein, $S$ is the region area, $L$ is the perimeter ${ }^{[6]}$ of the region. Setting the upper and lower threshold $C_{1}, C_{2}$, eliminating the region that $C>C_{1}$ and $C<C_{2}$.

(6) Testing the connected region of the processed image, marking the region where circular mark being located, then the position region that circular mark being located can be divided.

2.2 Image positing according to the geometric morphology

According to the region where the circular mark image that extracted in 2.1 being located, positing the circular mark on the image in combine with the geometric morphology of circular mark region. The position of circular mark on the image may be represented by the centroid of the circular mark region, using grey weight centroid algorithm to obtain the position coordinate ${ }^{[7]}$ of its centroid. Setting the grayscale of one circular mark $S$ as $I_{0}(x, y)$, and its centroid as $\left(x_{0}, y_{0}\right)$, according to grey weight centroid algorithm:

$$
\left\{\begin{array}{l}
x_{0}=\frac{\sum_{(x, y) \in S} x W(x, y)}{\sum_{(x, y) \in S} W(x, y)} \\
y_{0}=\frac{\sum_{(x, y) \in S} y W(x, y)}{\sum_{(x, y) \in S} W(x, y)}
\end{array}\right.
$$

Wherein,$W(x, y)$ is weight value, the grayscale value $I_{0}(x, y)$ may be taken as weight value, i.e.

$$
W(x, y)=I_{0}(x, y) \text { 。 }
$$

\section{Extracting experiment}

In order to make a contrast of the extracting effect between the above two methods, taking the image of circular mark collected in the lab as an example to conduct the extracting experiment.

\subsection{Least squares edge fitting extracting experiment based on Region of Interest}

The least squares edge fitting extracting experiment procedure and the extracting effect based on the Region of Interest are shown as Fig.2:

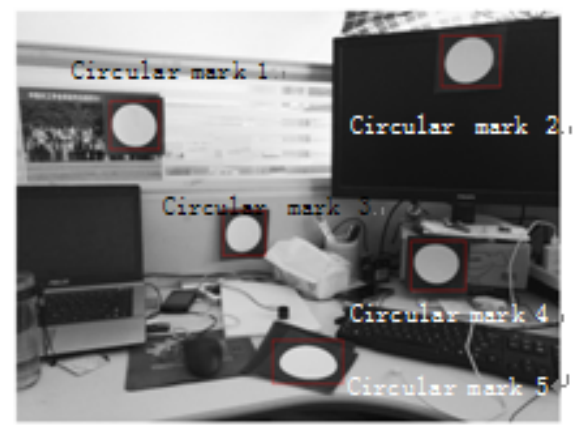

(a) The extracting of the region of interest

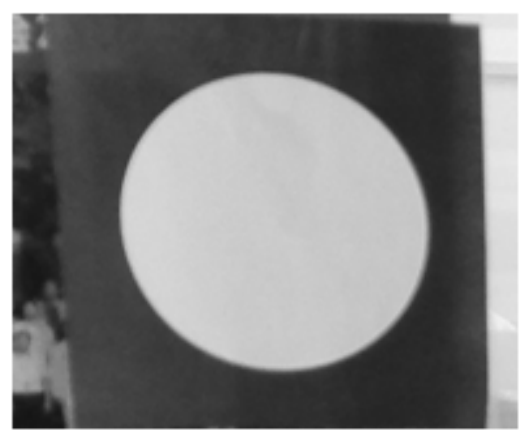

(b) The region of interest of circular mark1

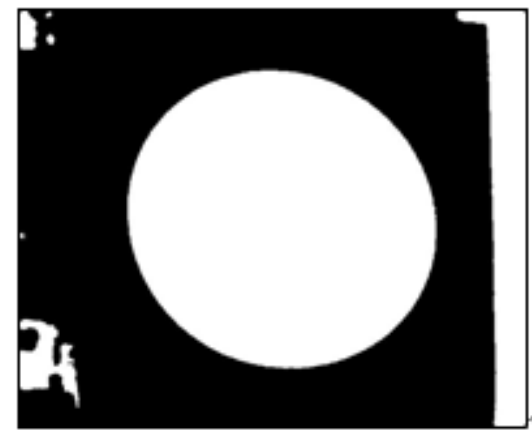

(c) The region of interest of circular mark1 


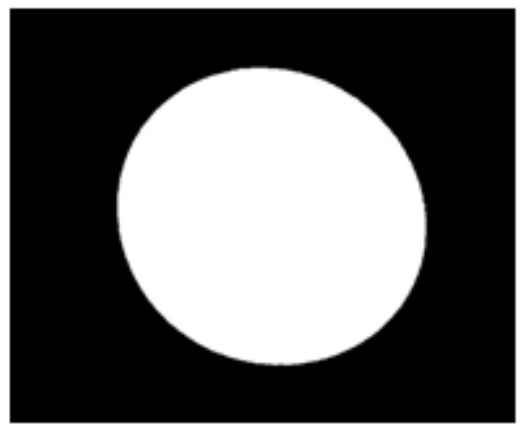

(d) Eliminating the jamming region

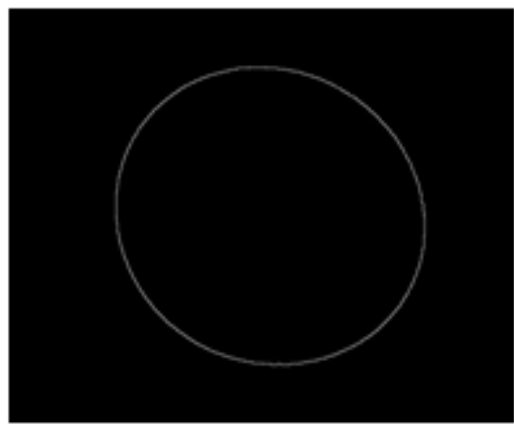

(e) Edge extracting

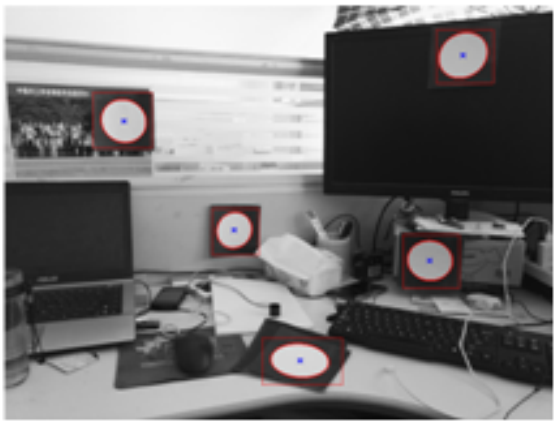

(f) The ellipse fitting and positing

Fig.2 Least squares edge fitting extracting experiment based on Region of Interest 4.2 The extracting experiment based on image gray scale and geometric morphology The extracting experiment procedure and its extracting effect based on the image gray scale and geometric morphology are shown as Fig.3:

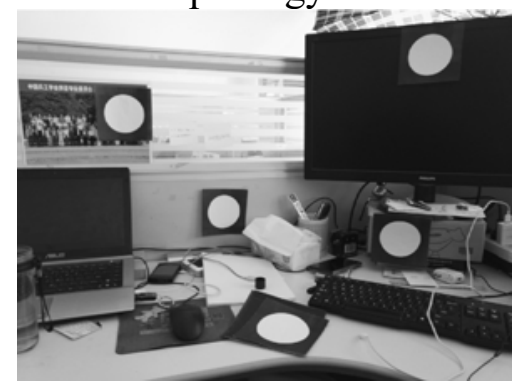

(a) Image filtering

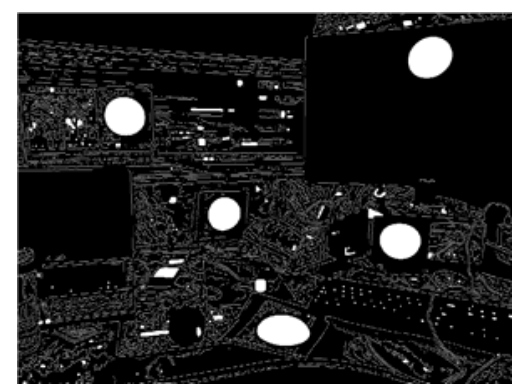

(d) cavity filling

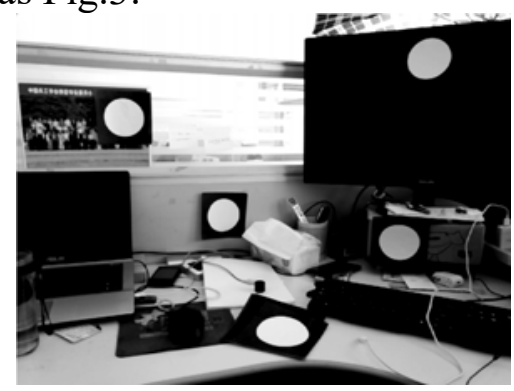

(b) grey level transformation

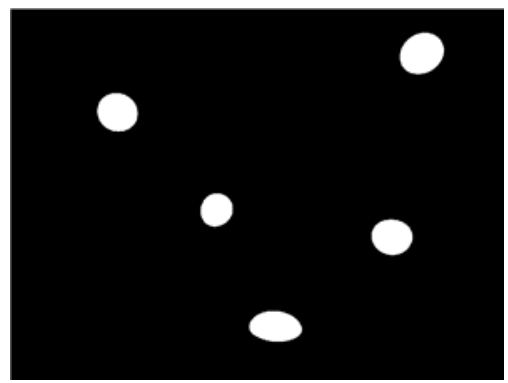

(e) eliminating the jamming area

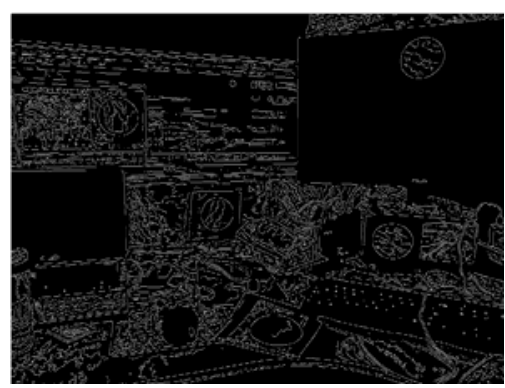

(c) edge testing

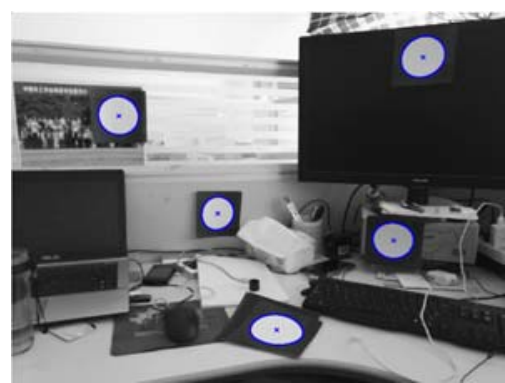

(f) circular mark positing

Fig. 3 The extracting experiment based on the image gray scale and geometric morphology

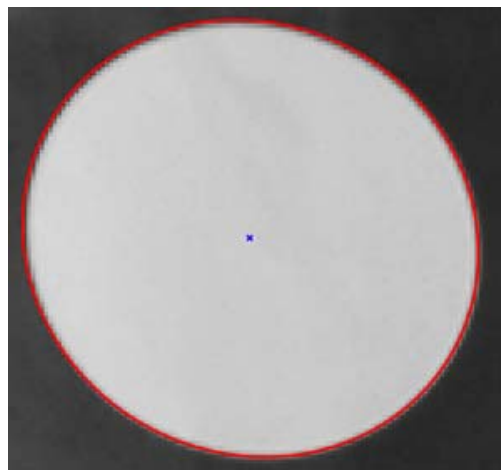

(a) The edge extracting effect of method 1

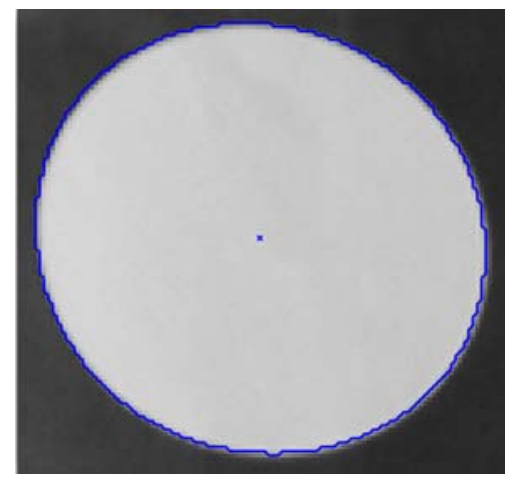

(b) The edge extracting effect of method 2

Fig.4 the contrast of edge extracting effect of circular mark1 


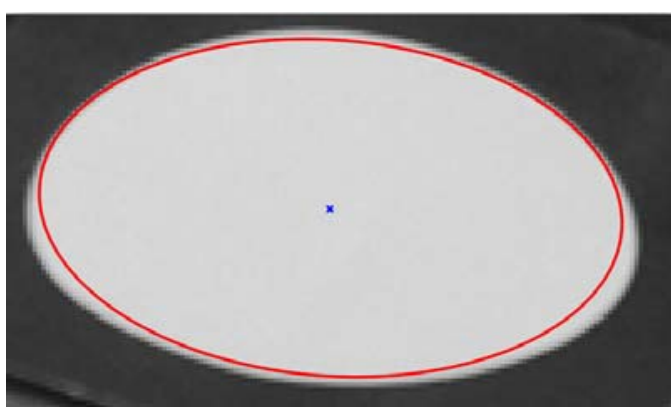

(a) The edge extracting effect of method 1

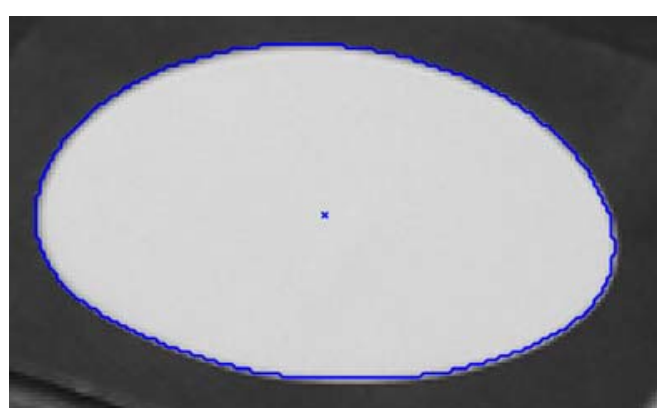

(b) The edge extracting effect of method 2

Fig. 5 the contrast of edge extracting effect of circular mark 5

\subsection{The contrast between the extracting results of circular mark}

\subsubsection{The contrast of the edge extracting effect}

The edge extracting effect of circular mark 1 and circular mark 5 that using the above two methods is shown as Fig4 and Fig5.

As seen from Fig.5(a), the extracted edge according to the method 1 is smooth ellipse, with better edge fitting effect, and the edge of the circular mark can be reflected clearly; as seen from Fig.5(a), the extracted edge according to the method 2 may reflect the true situation of the edges, however there are a few part of edge pixels will be mistaken as background owing to image segmentation etc. As can be seen from the contrast between Fig.5(a) and Fig.5(b), although regular ellipse may be extracted according to method 1, the extracted outline is in the interior of the circular mark, many pixel points of circular mark are mistaken as background, the effect of which is poor; while conducting the extracting of circular mark 5 according to the method 2, though the edges are not smooth enough, the actual edge of circular mark 5 can be able to be extracted, the circular mark may be divided from the background, with better effect in comparison with method 1 .

As the circular mark 1 is shooting nearly vertical, it will be imaged as a ellipse that rather regular; however as the circular mark 5 is shooting at large shooting angle, it will be imaged as a ellipse rather irregular. Therefore when using the least squares edge fitting method, there will be large difference between the extracting effect.

\subsubsection{The contrast between the positing results of circular mark}

According to the above two circular mark extracting experiment, the position of the obtained five circular marks on the image, the positing results of five circular marks according to the two methods and the demarcating deviation $\Delta s \quad\left(\Delta s=\sqrt{\left(x_{1}-x_{2}\right)^{2}+\left(y_{1}-y_{2}\right)^{2}}\right)$ of the two methods are shown as tablet 1 .

Table 1 The positing results of five demarcating circular marks according to the two methods

\begin{tabular}{cccccc}
\hline $\begin{array}{c}\text { circul } \\
\text { ar } \\
\text { mark }\end{array}$ & $\begin{array}{c}\text { The least squares edge } \\
\text { fitting method based on } \\
\text { the Region of Interest }\end{array}$ & $\begin{array}{c}\text { The method based on } \\
\text { image gray scale and } \\
\text { geometric morphology }\end{array}$ & deviation \\
\hline 1 & 277.96 & 268.94 & 277.78 & 268.81 & 0.22 \\
2 & 1067.58 & 115.73 & 1067.36 & 115.63 & 0.24 \\
3 & 534.63 & 523.17 & 534.58 & 522.91 & 0.26 \\
4 & 989.93 & 593.24 & 989.65 & 593.05 & 0.34 \\
5 & 687.57 & 826.52 & 687.28 & 826.1 & 0.51 \\
\hline
\end{tabular}

As can be seen from the tablet 1, the positing results of the circular mark according to the two methods are close to each other, when positing the circular mark 1, circular mark 2, and circular mark 3 with rather regular imaged ellipse shape, the largest positing deviation is 0.26 pixel; when positing 
the circular mark 4 and circular mark 5 with rather irregular imaged ellipse shape, the positing deviation is rather large, the largest deviation is 0.51 pixel.

\section{Summary}

This article studies two kind of circular mark extracting method, i.e. least squares edge fitting method based on the region of interest and method based on the image gray scale and geometric morphology, using the two methods to extract and position the 5 circular marks on the image, and making a contrast between the extracting effect and positing result according the two method.

As can be seen from the contrast of the edge extracting effect and the circular mark positing result, the least squares edge fitting method based on the region of interest can reduce the image processing computational complexity efficiently by extracting the region of interest, smooth ellipse edge that staying the same with the actual image of the circular mark may be obtained when the imaging of the circular mark is rather regular, however this method needs manual assistance, and the edge fitting effect will be bad when the circular mark image is irregular; the method based on the image gray scale and geometric morphology is on the basis of the image gray scale and geometric morphology of the circular mark, the outline of the circular mark may be depicted automatically according to the change of image gray scale in situations without the manual intervention, the edge extracting effect will not be effected by the image state of circular mark, however the edge extracted according to this method is serrated outline, the pixel point on the part of the circular mark is mistaken as background, there are certain deviations in comparison with the actual image situation of the circular mark at this point.

\section{References}

[1] Pei Yan, Wang Bin, Li Yuan. Improved Ellipse Fitting algorithm based on least squares method, Journal of Beijing University of Aeronautics and Astronautics, 34 (2008): 295-298.

[2] Chen Yan Xin, Qi FeiHu A new ellipse detection method based on random Hough transform, Journal of Infrared and Millimeter Waves, 19 (2000), 43-47.

[3] Xu L, Oja E. Randomized Hough Transform (RHT) : basic mechanisms, algorithms and computational complexities ,Computer, Vision Graphic Image Process: Image Understanding, 57 (1993): :131-154.

[4] C Huang, Q Liu, S Yu. Regions of interest extraction from color image based on visual saliency ,The Journal of Supercomputing, 58(2011): 20-33.

[5] Chen ZaiLiang The extraction method of region of interest of image, Changsha: Central South University, 2012.

[6] Liu XiangLei, Tong XiaoHua, Ma Jing video image sequence oval artificial measuring target rapid identification and tracking,Journal of Surveying and Mapping, 44 (2015): 663-669.

[7] Yu QiFeng, Lu Hongwei, Liu Xiaolin precision measurement and motion measurement based on the image [M]: Science Press, Beijing, 2002,pp. 120-122. 